\title{
Geoestatística como Metodologia para Estudar a Dinâmica Espaço-Temporal de Doenças Associadas a Colletotrichum spp. Transmitidos por Sementes
}

\author{
Marcelo C. Alves ${ }^{1}$, Edson A. Pozza ${ }^{2}$, José C. Machado², Dejânia V. Araújo², \\ Viviane Talamini² \& Marcelo S. Oliveira ${ }^{3}$
}

\begin{abstract}
1Departamento de Agricultura, e-mail: marcelocarvalhoalves@gmail.com; 리 Departamento de Fitopatologia, e-mail: eapozza@ufla.br; ${ }^{3}$ Departamento de Ciências Exatas, Universidade Federal de Lavras, Cx. Postal 3037, CEP 37200-000, Lavras, MG, e-mail: marcelo.oliveira@ufla.br
\end{abstract}

(Aceito para publicação em 12/10/2006)

Autor para correspondência: Edson Ampélio Pozza

\begin{abstract}
ALVES, M.C., POZZA, E.A., MACHADO, J.C., ARAÚJO, D.V., TALAMINI, V. \& OLIVEIRA, M.S. Geoestatística como metodologia para estudar a dinâmica espaço-temporal de doenças associadas a Colletotrichum spp. transmitidos por sementes. Fitopatologia Brasileira 31:557-563. 2006.
\end{abstract}

\section{RESUMO}

Objetivou-se no presente trabalho caracterizar o progresso espaço-temporal da severidade da antracnose do feijoeiro comum e da ramulose do algodoeiro por meio da Geoestatística. Os experimentos foram conduzidos no campo, durante o período das águas, em diferentes épocas. Sementes inoculadas pelo método da restrição hídrica foram semeadas no centro de parcelas experimentais constituindo fonte de inóculo do tipo ponto. Foram realizadas semanalmente seis avaliações da severidade das doenças com base em escalas de notas. Pelos modelos de semivariograma isotrópicos esféricos e gaussianos ajustados aos dados, verificou-se o padrão de distribuição agregado e a dependência espacial de ambos os patossistemas. Com o mapeamento da severidade das doenças pelo método da krigagem ordinária em blocos, verificou-se menor severidade das doenças nos primeiros estádios, com aumento gradual ao longo do tempo. A antracnose apresentou formação inicial de focos de inóculo secundário, além do foco com inóculo inicial, que coalesceram com o tempo. A severidade da ramulose diminuiu gradualmente de forma radial e contínua do centro da parcela para as extremidades, com maior capacidade de infecção de plantas vizinhas, quando comparada à antracnose, possivelmente pela maior agressividade do patógeno e hábito de crescimento arbustivo do algodoeiro.

Palavras-chave adicionais: Colletotrichum lindemuthianum, Colletotrichum gossypii var. cephalosporioides, krigagem ordinária.

\begin{abstract}
Geoestatistics as methodology to study the space-time dynamics of diseases transmitted by seed-borne Colletotrichum spp.

The aim of this work was the characterization of the space-time severity progress of the common bean Anthracnose and cotton Ramulose through Geoestatistics. The experiments were conducted over different periods during the rainy season, in field conditions. Seeds inoculated by the water restriction method were sowed in the center of experimental plots standing as inoculum source of point type. Six evaluations of the epidemics' severity were done weekly following disease diagrams. The clustered pattern and the spatial dependence in both pathosystems were verified with isotropic spherical and gaussian semivariogram models adjusted to the data. With the ordinary block kriging method, it was possible to verify that the severity of the diseases was lower in the first stages, becoming higher over time in both epidemics. Anthracnose presented initially secondary inoculum focus formation besides the focus with initial inoculum with later coalescence. The severity of Ramulose decreased gradually, both radially and smoothly from the center of the plot to the borders, with a larger capacity to infect neighboring plants, when compared to the Anthracnose, possibly due to the fungi's aggressiveness and to the cotton plant's shrubby growth habit.

Additional keywords: Colletotrichum lindemuthianum, Colletotrichum gossypii var. cephalosporioides, ordinary kriging.
\end{abstract}

\section{INTRODUÇÃO}

A semente é um meio de disseminação e sobrevivência de fitopatógenos por longos períodos de tempo. Patógenos associados a sementes podem reduzir a germinação, o vigor, deteriorá-las em armazéns, introduzir patógenos em novas áreas e aumentar o inóculo em lavouras já estabelecidas. Se o inóculo primário, transportado via-semente, encontrar ambiente favorável e o hospedeiro for suscetível, pode-se estabelecer focos iniciais de infecção. A partir daí poderá 
ocorrer o progresso da doença no espaço e no tempo, devido ao inóculo secundário, produzido nas lesões das plantas infectadas (Campbell \& Madden, 1990; Tanaka \& Menten, 1992; Talamini et al., 2001).

Dessa forma, conhecer o progresso de uma doença ao longo do tempo e do espaço, a partir da fonte de inóculo, possibilita conhecer a dinâmica da interação de patógenos com hospedeiros, auxilia a estabelecer padrões de tolerância para patógenos em sementes, contribui para o manejo da doença e conseqüente aumento da produtividade das culturas (Silva et al., 2000; Talamini et al., 2001; Bergamin Filho et al., 2004). A disseminação do patógeno e o progresso da doença ao longo do espaço são dependentes do arranjo das plantas, da intensidade da doença na fonte de inóculo e da sua forma de disseminação (Campbell \& Madden, 1990). Entretanto, em alguns métodos para caracterizar padrões espaciais de doenças de plantas, não se consideram a dependência espacial entre a intensidade da doença em um indivíduo e a sua vizinhança. Como exemplo citam-se as análises estatísticas descritivas de 'quadrats', testes de 'run' e 'doublet', índice VM (variância/média) e lei de Taylor (Bergamin Filho et al., 2004; Campbell \& Madden, 1990). Seria então necessário utilizar metodologias estatísticas capazes de considerar a distância entre amostras para quantificar e representar a dependência espacial com estimação ótima, conforme é preconizado na teoria das variáveis regionalizadas, base dos fundamentos da geoestatística (Silva et al., 2000).

A geoestatística tem sido aplicada para modelar o padrão espaço-temporal e gerar hipóteses sobre aspectos epidemiológicos de doenças de plantas (Chellemi et al., 1988; Larkin et al., 1995; Nelson et al., 1999; Wallace \& Hawkins, 1994; Jaime-Garcia et al., 2001; Charest et al., 2002) devido ao padrão agregado do inóculo e da doença. Entre os patógenos associados a doenças com esse padrão encontram-se os de espécies do gênero Colletotrichum (Machado et al., 2002; Bianchini et al., 2005). Diversas espécies de Colletotrichum, entre elas Colletotrichum lindemuthianum (Sacc. \& Magnus) Briosi \& Cavara e Colletotrichum gossypii (South) var. cephalosporioides A.S. Costa, ambos encontram-se associados a sementes de seus hospedeiros e são potencialmente transmitidos por essa via (Richardson, 1990; Tanaka \& Menten, 1988; Pinto et al., 2001; Talamini et al., 2001; Araújo, 2004; ). Araújo (2004) e Pinto et al. (2001) utilizaram os métodos baseados em mapas de 'quadrats' e análise 'ordinary runs` para descrever o padrão de disseminação desses fungos a partir de semente, respectivamente. Entretanto, nesses métodos não se considera a distância entre as observações e, muitas vezes, as interpretações sobre os patossistemas são limitadas. Dessa forma, objetivou-se com o presente trabalho caracterizar a estrutura e a magnitude da dependência espacial e mapear a severidade da antracnose (Colletotrichum lindemuthianum) do feijoeiro (Phaseolus vulgaris L.) e da ramulose (Colletotrichum gossypii var. cephalosporioides) do algodoeiro (Gossypium hirsutum L.) por meio da Geoestatística.

\section{MATERIAL E MÉTODOS}

Os dados analisados neste trabalho foram cedidos por Talamini (2003) e Araújo (2004). Ambos os trabalhos tinham sido conduzidos na área experimental do Departamento de Fitopatologia (DFP) da Universidade Federal de Lavras (UFLA).

\section{Experimento com a antracnose do feijoeiro}

O experimento foi conduzido durante o período das águas (dezembro/98 a março/99) em altitude de 918 $\mathrm{m}$, latitude sul de $21^{\circ} 14^{\prime}$, longitude oeste de $45^{\circ} 00^{\prime}$ em Latossolo Roxo. Antes de instalar o experimento foram retiradas amostras de solo pelas quais foram realizadas as recomendações de correção e adubação. Realizou-se adubação no sulco de plantio, na proporção de $500 \mathrm{~kg} / \mathrm{ha}$ da formulação 4-14-8 de N-P-K, e de cobertura com $150 \mathrm{~kg} / \mathrm{ha}$ de sulfato de amônio, aos 25 dias após a semeadura.

Foram selecionados lotes de sementes suscetíveis, da cultivar Carioca, livres de infecção por $C$. lindemuthianum. Para a seleção das sementes foi utilizado o método do rolo de papel (Machado et al., 2002). Duas sementes inoculadas artificialmente foram consideradas fonte tipo ponto $(0,5 \%$ das sementes utilizadas) (Campbell \& Madden, 1990) e em seguida, semeadas exatamente no meio de uma parcela constituída por 9 linhas, com $5 \mathrm{~m}$ de comprimento, sendo consideradas como área útil 7 linhas de 4 metros, no espaçamento de $0,5 \mathrm{~m}$ entre linhas com 12 plantas $/ \mathrm{m}$. Utilizaram-se como bordadura 4 fileiras de milho semeadas 30 dias antes do início do experimento.

As sementes foram inoculadas pelo método da restrição hídrica, desenvolvido por Carvalho et al. (1998). Sementes de feijão foram mantidas por 7 dias em meio de cultura agarizado com $C$. lindemuthianum raça 89 , em potencial osmótico de $-1,0 \mathrm{MPa}$, a temperatura de $20^{\circ} \mathrm{C}$, com $12 \mathrm{~h}$ de luz e $12 \mathrm{~h}$ de escuro em estufa BOD.

Antes do plantio, as sementes não inoculadas foram tratadas com benomil + thiram. Capinas, irrigação por aspersão (40 mm em duas aplicações por semana) e aplicações de inseticidas e fungicidas para o controle de outras doenças que não a antracnose (oxicarboxim) foram realizadas sempre que necessárias. Para o controle de plantas daninhas foram realizadas capinas manuais.

O estádio vegetativo do feijoeiro foi avaliado com base na escala de Fernandez et al. (1986). As avaliações de doença foram feitas semanalmente, iniciando-se 26 dias após o plantio (DAP) até 61 DAP, observando-se todas as plantas da área útil das parcelas. A severidade em cada avaliação foi determinada avaliando-se todas as folhas de cada planta com sintomas, utilizando a escala de avaliação para antracnose do feijoeiro (Tamayo, 1995), onde: $1=0 \% ; 3=1 \% ; 5=5 \%$; $7=10 \%$ e, $9=25 \%$ da área foliar lesionada.

\section{Experimento com a ramulose do algodoeiro}

O experimento foi instalado em dezembro de 2002 a julho de 2003, em altitude de 921 metros, 21 $1^{\circ} 13$ '50"de 
latitude sul e $45^{\circ} 58^{\prime} 41^{\prime \prime}$ de longitude oeste, em solo com textura argilosa. As adubações foram realizadas com base nos resultados de análise química e física do solo. Utilizaram-se $669 \mathrm{Kg} / \mathrm{ha}$ da mistura 20-90-40 (N-P $\mathrm{O}_{5}$ $\mathrm{K}_{2} \mathrm{O}$ ), tendo como fontes sulfato de amônio, superfosfato simples e cloreto de potássio. A adubação de cobertura foi realizada em duas etapas, aos 30 e 45 dias após o plantio, na proporção de $184 \mathrm{Kg} / \mathrm{ha}$ da mistura 30-0-20. A parcela experimental constitui-se por seis linhas de cinco metros de comprimento. $\mathrm{O}$ espaçamento foi de 0,9 metros entre linhas, com 11 sementes por metro linear.

Foram utilizadas sementes da linhagem IAC 01/273 da safra 2001/02, altamente suscetíveis à ramulose, livres de infecção por C. gossypii var. cephalosporioides. Para a seleção das sementes, foi utilizado o teste de sanidade, método de incubação em papel de filtro, umedecido com água destilada esterilizada contendo 2,4-D na concentração de 10 ppm (Machado et al., 2002) e teste de germinação realizado segundo regras para análise de sementes (Brasil, 1992). Após deslintadas, $2 \%$ das sementes foram inoculadas com cultura pura de C. gossypii var. cephalosporioides cultivada em BDA por sete dias, pelo método descrito por Carvalho et al. (1998) em potencial hídrico de -1 MPa e semeadas no centro da parcela, constituindo fonte tipo ponto (Campbell \& Madden, 1990).

O desbaste foi realizado aos 30 dias após a semeadura, com o objetivo de manter 30 plantas por linha. As avaliações de severidade foram feitas aos 37, 44, 51, 58, 65 e, 80 dias após o plantio (DAP), por meio da escala de notas elaborada por Costa (1941), em que: 0 = Ausência de sintomas; $1=$ Lesões necróticas estreladas nas folhas localizadas no ápice da planta; 2 = Encurtamento dos internódios no ápice da planta; 3 = Superbrotamento acentuado, redução no crescimento da planta e, $4=$ Planta com superbrotamento e desenvolvimento comprometido, morte das partes afetadas.

\section{Análise Geoestatística dos dados}

A dependência espacial foi analisada por meio de ajustes de semivariogramas, com base na pressuposição de estacionariedade da hipótese intrínseca. A análise Geoestatística dos dados foi realizada com os valores das notas de severidade obtidos nas diferentes épocas de avaliação. O semivariograma experimental foi estimado por (Burrough \& McDonnell, 1998):

$$
\hat{\gamma}(h)=\frac{1}{2 N(h)} \sum_{i=1}^{N(h)}\left[Z\left(x_{i}\right)-Z\left(x_{i}+h\right)\right]^{2}
$$

em que,

$$
\begin{aligned}
& \gamma(h)=\text { Semivariância estimada; } \\
& N(h)=\text { Número de pares de observações } \\
& Z\left(x_{i}\right), Z\left(x_{i}+h\right) \text { separados pela distância } h .
\end{aligned}
$$

Os modelos teóricos de semivariogramas isotrópicos foram estimados por (Burrough \& McDonnell, 1998):

Modelo Esférico

$$
\gamma(h)= \begin{cases}C_{o}+C\left\{\frac{3 h}{2 a}-\frac{1}{2}(h / a)^{3}\right\} & \text { para } 0<\mathrm{h}<\mathrm{a} \\ C_{o}+C & \text { para } \mathrm{h} \geq \mathrm{a}\end{cases}
$$

Modelo Gaussiano:

$$
\gamma(h)=C_{o}+C\left\{1-\exp \left(-h^{2} / a^{2}\right)\right\} \text { para } \mathrm{h}>\mathrm{a}
$$

Em que ' $\mathrm{C}_{0}$ ' é o efeito pepita e ' $\mathrm{C}_{0}+\mathrm{C}^{\prime}$ o patamar, 'a' o alcance e 'h' a distância.

$\mathrm{O}$ ajuste dos modelos foi escolhido em função dos parâmetros dos semivariogramas, os coeficientes de determinação $\left(\mathrm{R}^{2}\right)$, a soma de quadrados do resíduo e a autovalidação ('Jack-Knife'). Os parâmetros dos semivariogramas foram representados nos gráficos pelo efeito pepita $(\mathrm{Co})$, patamar $(\mathrm{Co}+\mathrm{C})$ e alcance (Ao). Para a análise do grau de dependência espacial das variáveis, foi utilizada a classificação de Cambardella et al. (1994), em que são considerados de forte dependência espacial os semivariogramas que têm efeito pepita $25 \%$ do patamar, moderada quando entre 25 e $75 \%$ e de fraca quando $>75 \%$.

Após o ajuste dos semivariogramas, foi realizada interpolação por krigagem ordinária em blocos $2 \times 2$ para mapear as epidemias. O número de vizinhos mais próximos para a estimação variou em função da autovalidação, situando-se entre 10 e 16 vizinhos. A krigagem é uma técnica utilizada na Geoestatística com o objetivo de estimar valores de variáveis para locais onde essas variáveis não foram medidas com base em valores adjacentes interdependentes. Para utilizar esse método e obter acurácia e precisão satisfatória na estimativa é necessário existir dependência espacial definida pelo semivariograma (Salviano, 1996).

$\mathrm{O}$ valor médio de $\mathrm{z}$ em um bloco $\mathrm{B}$ foi estimado por (Burrough \& McDonnell, 1998):

$$
z(B)=\sum \lambda_{i} \cdot z\left(x_{i}\right)
$$

Em que, $\mathrm{z}(\mathrm{B})$ é o valor estimado da severidade da doença no bloco, $\lambda_{i}$ é o vetor de pesos determinado pelo ajuste do semivariograma, e, $z\left(x_{i}\right)$ é o valor da variável $z$ representada por vizinhos de um bloco $B$.

\section{RESULTADOS E DISCUSSÃO}

Pelo método de infecção da semente, descrito por Carvalho et al. (1998), garantiu-se a eficiência na transmissão da antracnose no feijoeiro e da ramulose no algodoeiro da semente para as plantas no campo.

A dependência espacial e o padrão agregado de dispersão das epidemias foram constatados por meio 
dos modelos variográficos. Ajustaram-se modelos de semivariograma isotrópico esférico para a antracnose dos 26 aos 54 DAP e gaussiano, em todas as épocas de avaliação para a ramulose e aos 61 DAP para a antracnose (Tabela 1). Os semivariogramas atingiram um patamar dentro de cada período de avaliação, correspondente ao seu alcance, de forma a satisfazer as suposições de estacionariedade (Lamparelli et al., 2001).

Houve aumento dos alcances e dos patamares dos semivariogramas para ambas as doenças, com as épocas de avaliação, correspondente ao aumento do progresso espacial das doenças no campo, com o passar do tempo, devido ao aumento da severidade e do número de plantas doentes no foco primário. Os valores de alcance para antracnose do feijoeiro variaram de 1,63 metros, aos 26 DAP, até 3,15 metros, aos 61 DAP e, para a ramulose do algodoeiro, de 5,19, aos 37 DAP, até 6,82 metros, aos 80 DAP, indicando a distância máxima de progresso das doenças em cada período avaliado (Tabela 1). De forma semelhante à verificada no estudo de Larkin et al. (1995), a força do grau de dependência espacial também aumentou com o tempo para a ramulose e, para a antracnose, de 26 aos 54 DAP. Isso implica no fato de, cada vez mais, plantas sadias ao lado de plantas doentes tornarem-se doentes, com o passar do tempo. A força do grau de dependência espacial na última avaliação da antracnose (61DAP) diminuiu, entretanto, isso ocorreu em função do ajuste do modelo gaussiano em detrimento do modelo esférico. Entretanto, de acordo com a classificação de Cambardella et al. (1994), a antracnose apresentou forte grau de dependência espacial entre plantas em todas as épocas de avaliação e, a ramulose apresentou grau de dependência espacial moderada aos 37 e 44 (DAP) e forte grau de dependência espacial em 51, 58, 65 e 80 DAP (Tabela 1).

Araújo (2004) caracterizou o padrão espacial da ramulose do algodoeiro como agregado, pelo método baseado em mapas de 'quadrats'. Apesar de ser uma técnica simples, de baixo custo, e fornecer uma visualização rápida de padrões (Campbell \& Madden, 1990), esse tipo de mapeamento não considerou a distância entre amostras e a escala real da parcela estudada, causando incerteza sobre o progresso espacial da doença. Além disso, modelos matemáticos e algoritmos de interpolação não foram utilizados para extrair mais informações sobre a provável distribuição doença. Pinto et al. (2001) caracterizou o padrão espacial da antracnose do feijoeiro pela análise 'ordinary runs'. Verificou-se padrão agregado no plantio das águas e das secas, entretanto, nas duas primeiras avaliações do plantio das secas, constatouse padrão casualizado, possivelmente, por esse método não considerar a distância entre as amostras. Além disso, segundo Campbell \& Madden (1990), esse método é melhor aplicado a dados binários, sendo que o número de amostras pode influenciar em sua eficiência.

Por meio dos mapas de krigagem observou-se menor variabilidade espacial das epidemias nos primeiros estádios, com aumento gradual até o último tempo de avaliação (Figura 1). A krigagem ordinária em blocos foi escolhida por gerar uma superfície mais suavizada quando comparada a krigagem ordinária pontual, já que representa o valor médio de uma área ao redor do ponto estimado (Burrough \& McDonnell, 1998). Tanto a krigagem em blocos quanto a pontual podem ser utilizadas no mapeamento de doenças de plantas. Entretanto, nos mapas produzidos pela krigagem em blocos observou-se superfície mais suavizada, principalmente, em situações em que o efeito pepita é maior (Wallace \& Hawkins, 1994).

Com os mapas de krigagem pôde-se constatar a menor variabilidade espacial da severidade nos primeiros estádios da doença, com aumento gradual até o último tempo de avaliação e o padrão agregado das epidemias, com maior

TABELA 1 - Coeficientes e parâmetros de semivariogramas isotrópicos relativos à severidade da antracnose do feijoeiro e ramulose do algodoeiro em diferentes épocas de avaliação

\begin{tabular}{|c|c|c|c|c|c|c|c|c|}
\hline \multirow[b]{2}{*}{ Doença } & \multirow[b]{2}{*}{$\begin{array}{c}\text { Dias } \\
\text { Após } \\
\text { plantio }\end{array}$} & \multirow[b]{2}{*}{ Modelo } & \multicolumn{4}{|c|}{ Parâmetros } & \multicolumn{2}{|c|}{ Coeficientes } \\
\hline & & & $\begin{array}{c}\text { Efeito pepita } \\
\text { (Co) }\end{array}$ & $\begin{array}{c}\text { Patamar } \\
(\mathrm{Co}+\mathrm{C})\end{array}$ & $\begin{array}{c}\text { Alcance } \\
\text { (a) }\end{array}$ & $\begin{array}{c}\text { Alcance } \\
\text { prático }\end{array}$ & $\begin{array}{l}R^{2} \\
(\%)\end{array}$ & $\begin{array}{r}\mathrm{Co} / \\
\mathrm{Co}+\mathrm{C}\end{array}$ \\
\hline \multirow[t]{6}{*}{ Antracnose } & 26 & Esférico & 0,05 & 0,28 & 1,63 & 1,63 & 99,10 & 0,18 \\
\hline & 33 & Esférico & 0,05 & 0,74 & 1,68 & 1,68 & 99,90 & 0,07 \\
\hline & 40 & Esférico & 0,04 & 0,98 & 1,72 & 1,72 & 99,70 & 0,04 \\
\hline & 47 & Esférico & 0,03 & 1,28 & 1,76 & 1,76 & 99,40 & 0,02 \\
\hline & 54 & Esférico & 0,00 & 1,76 & 1,90 & 1,90 & 98,60 & 0,00 \\
\hline & 61 & Gaussiano & 0,37 & 3,17 & 1,81 & 3,15 & 99,50 & 0,11 \\
\hline \multirow[t]{6}{*}{ Ramulose } & 37 & Gaussiano & 0,18 & 0,39 & 2,99 & 5,19 & 99,20 & 0,46 \\
\hline & 44 & Gaussiano & 0,35 & 0,77 & 3,10 & 5,37 & 99,50 & 0,46 \\
\hline & 51 & Gaussiano & 0,32 & 1,39 & 3,57 & 6,18 & 99,30 & 0,23 \\
\hline & 58 & Gaussiano & 0,39 & 1,72 & 3,58 & 6,21 & 98,50 & 0,22 \\
\hline & 65 & Gaussiano & 0,54 & 2,68 & 3,59 & 6,23 & 97,60 & 0,20 \\
\hline & 80 & Gaussiano & 0,74 & 3,93 & 3,94 & 6,82 & 98,00 & 0,18 \\
\hline
\end{tabular}


Geoestatística como metodologia para estudar a dinâmica...

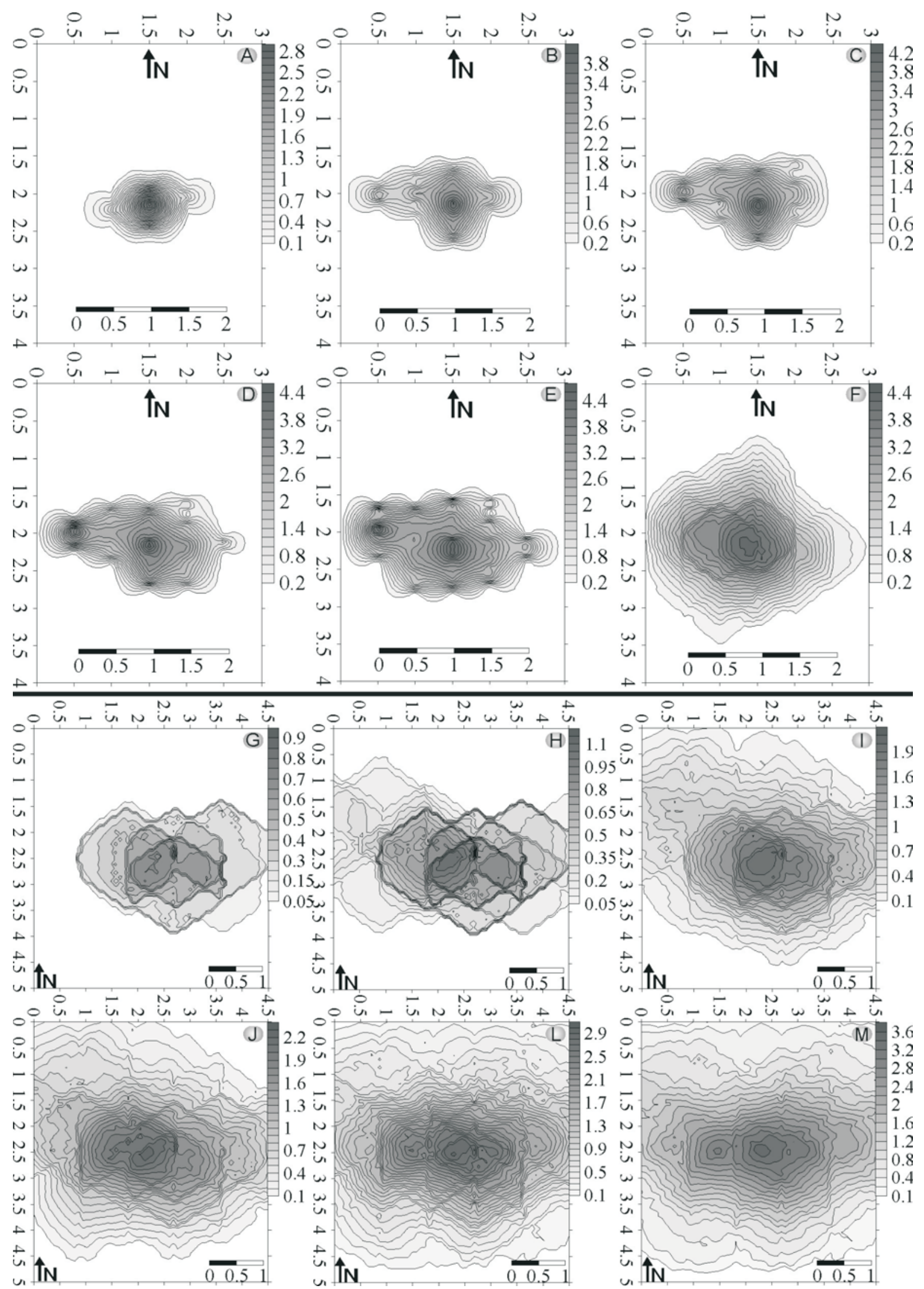

FIG. 1 - Mapas resultantes da krigagem ordinária em blocos, da severidade da antracnose do feijoeiro em 26 (a), 33 (b), 40 (c), 47 (d), 54 (e) e, 61 dias após o plantio (DAP) (f); e, da severidade da ramulose do algodoeiro em 37 (g), 44 (h), 51 (i), 58 (j), 65 (l) e, 80 (DAP) (m). 
severidade ao redor da fonte de inóculo inicial do tipo ponto. Entretanto, observando-se os mapas, pôde-se constatar que a antracnose apresentou mecanismo de dispersão com a formação de focos de inóculo secundário além do foco primário, representados pelas isolinhas nos mapas, aos 33, 40, 47 e 54 DAP, indicando focos com maior severidade da doença distantes da fonte de inóculo primário tipo ponto. Posteriormente, aos 61 DAP, os focos acabaram por coalescer e a disseminação da doença nesse período apresentou padrão de disseminação contínuo e radial, com maior severidade no centro da parcela, próximo à fonte de inóculo primário tipo ponto (Figura 1). Essa estratégia de disseminação do patógeno ocorre, provavelmente, para satisfazer processos biológicos fundamentais, como reprodução, dispersão, competição, sobrevivência, entre outros, gerando um maior número de plantas infectadas em focos distantes da fonte (Fernandez, 1981). Segundo Bergamin Filho et al. (2004), essa dinâmica populacional é comumente verificada em doenças cujos agentes etiológicos são transmitidos por vetores, como por exemplo, na tristeza, causada pelo Citrus tristeza virus.

Para a ramulose do algodoeiro, observou-se nos mapas maior severidade da doença ao redor da fonte de inóculo primário. A severidade da doença diminuiu gradualmente, de forma radial, do centro da parcela para as extremidades. Com os modelos gaussianos ajustados ao progresso espaçotemporal da ramulose foi possível constatar a grande capacidade de disseminação do patógeno em curto período de tempo e a alta continuidade espacial com propagação radial, ou seja, a ocorrência da doença em uma planta implicou a infecção de plantas vizinhas até determinado limite devido ao tempo da epidemia. A disseminação da doença foi mais intensa a partir dos 51 DAP, estabilizandose aos 80 DAP, sendo que a formação de focos secundários com alta severidade pôde ser observada aos 65 e 80 DAP (Figura 1). Santos et al. (1994) também observaram a rápida disseminação da ramulose. Esses autores estudando o progresso espacial da ramulose, a partir de plantas inoculadas, detectaram a doença em plantas adjacentes à fonte de inóculo aos 32 DAP e a maior incidência nas plantas localizadas mais próximas (1-2m) da fonte do inóculo inicial, entretanto, nenhuma metodologia estatística foi empregada e o padrão da doença não foi quantificado. Outras vantagens da metodologia de análise Geoestatística com relação aos métodos utilizados por Araújo (2004), Pinto et al. (2001) e Santos et al. (1994) incluem a possibilidade de quantificar a magnitude e o grau de dependência espacial, mapear a variabilidade espacial das epidemias com um interpolador exato e preciso, bem como visualizar o progresso espacial ao longo do tempo. Além disso, os modelos de semivariogramas obtidos podem ser utilizados em estudos posteriores para predizer a probabilidade de ocorrência das epidemias em campos naturalmente infectados e, melhorar as estratégias de amostragem sob diferentes escalas. Com os mapas de krigagem também foi possível constatar a transmissibilidade dos patógenos da semente infectada para as plântulas e, posteriormente, entre plantas no campo, ao longo do tempo e, conseqüentemente, a natureza policíclica das doenças.

\section{REFERÊNCIAS BIBLIOGRÁFICAS}

ARAÚJO, D.V. Níveis de inóculo de Colletotrichum gossypii var. cephalosporioides nas sementes e sua influencia na epidemia da Ramulose do algodoeiro. 2004. Dissertação de Mestrado. Lavras MG. Universidade Federal de Lavras. 2004.

BERGAMIN FILHO, A., HAU, B., AMORIM, L., \& JESUS JUNIOR, W.C. Análise espacial de epidemias. In: Vale, F.X.R., Jesus Junior, W.C. \& Zambolim, L. (Eds.) Epidemiologia aplicada ao manejo de doenças de plantas. Belo Horizonte: Editora Perffil, 2004. pp. 193-240.

BIANCHINI, A., MARINGONI, A.C. \& CARNEIRO, S.M.T.P.G. Doenças do feijoeiro (Phaseolus vulgaris). In: Kimati, H., Amorim, L., Rezende, J.A.M., Bergamin Filho, A. \& Camargo, L.E.A. (Eds.) Manual de Fitopatologia. São Paulo. Editora Agronômica Ceres, v.2, 2005. pp. 333-349.

BRASIL, Ministério da Agricultura. Regras para análise de sementes. Brasília: LANARV/SNAD/MA, 1992.

BURROUGH, P.A. \& McDONNELL, R.A. Principles of geographical information systems. 2nd Edition. Oxford. Oxford University Press, 1998.

CAMBARDELLA, C.A., MOORMAN, T.B., NOVAK, J.M., PARKIN, T.B., KARLEN, D.L., TURCO, R.F. \& KONOPKA, A.E. Fieldscale variability of soil properties in central iowa soils. Soil Science Society of America Journal 58:1501-1511. 1994.

CAMPBELL, C.L. \& MADDEN, L.V. Introduction to plant disease epidemiology. New York. J. Wiley \& Sons. 1990.

CARVALHO, J.C.B., MACHADO, J.C. \& VIEIRA, M.G.G.C. Desenvolvimento de metodologia de infecção de sementes de feijão por Colletotrichum lindemuthianum através do condicionamento osmótico. Fitopatologia Brasileira (Suppl.) 23:232. 1998.

CHAREST, J., DEWDNEY, M., PAULITZ, T., PHILION, V. \& CARISSE, O. Spatial distribution of Venturia inaequalis airborne ascospores in orchards. Phytopathology, 92:769-779. 2002.

CHELLEMI, D.O., ROHRBACH, K.G., YOST, R.S. \& SONODA, R.M. Analysis of the spatial pattern of plant pathogens on diseased plants using geoestatistics. Phytopathology 78:221-226. 1988.

COSTA, A.S. Investigação sobre a ramulose. Relatório. Campinas: Instituto Agronômico de Campinas, Seção de Algodão, 1941. (relatório, 1012).

FERNANDEZ, C.M.A. Influência do número de focos de inóculo inicial de Colletotrichum lindemuthianum (Sacc. \& Magn.) Scrib. no desenvolvimento da antracnose em folhas, vagens e sementes de Phaseolus vulgaris L. 1981. (Dissertação em Fitopatologia) Universidade Federal de Viçosa, MG.

FERNANDEZ, F., GEPTS, P. \& LOPEZ, M. Etapas de desarollo de la planta de fríjol común (Phaseolus vulgaris L.). Cali, Colômbia: CIAT, 1986.

JAIME-GARCIA, R., ORUM, T.V., FELIX-GASTELUM, R., TRINIDAD-CORREA, R., VANETTEN, H.D. \& NELSON, M.R. Spatial analysis of Phytophthora infestans genotypes and late 
blight severity on tomato and potato in the del fuerte valley using geostatistics and geographic information systems. Phytopathology 91:1156-1165. 2001.

JAIME-GARCIA, R. \& COTTY, P.J. Aflatoxin contamination of commercial cottonseed in south Texas. Phytopathology 93:11901200. 2003.

LAMPARELLI, R.A.C., ROCHA, J.V. \& BORGHI, E. Geoprocessamento e agricultura de precisão: fundamentos e aplicações. Guaíba. Agropecuária. 2001.

LARKIN, R.P., GUMPERTZ, M.L. \& RISTAINO, J.B. Geostatistical analysis of Phytophthora epidemic development in commercial bell pepper fields. Phytopathology 85:191-203. 1995.

MACHADO, J.C., LANGERAK, C.J. \& JACCOUD-FILHO, D.S. Seed-borne fungi: a contribution to routine seed health analysis. Zurich. International Seed Testing Association. 2002.

NELSON, M.R., ORUM, T.V., JAIME-GARCIA, R. \& NADEEM, A.Applications of geographic information systems and geostatistics in plant disease epidemiology and management. Plant Disease 83:308-319 1999 .

PINTO, A.C.S., POZZA, E A., TALAMINI, V., MACHADO, J.C., SALES, N.L.P., GARCIA JÚNIOR, D. \& SANTOS, D.M. Análise do padrão espacial e do gradiente da antracnose do feijoeiro em duas épocas de cultivo. Summa Phytopathologica 27:392-398. 2001.

RICHARDSON, M.J. An annotated list of seed-borne diseases. 4 ed. Zurich. International Seed Testing Association. 1990.

SALVIANO, A.A.C. Variabilidade de atributos de solo e de Crotalaria juncea em solo degradado do município de PiracicabaSP. Piracicaba, 1996. Tese (Doutorado) - Escola Superior de Agricultura "Luiz de Queiroz", Universidade de São Paulo.

SANTOS, G.R., ZAMBOLIM, L., VALE, F.X.R., MAFFIA, L.A. \& VIEIRA, J.M. Progresso e gradiente da ramulose do algodoeiro.
Fitopatologia Brasileira 19:390-393. 1994.

SILVA, E.A., SILVA, J.F.V., MONICO, J.F.G. \& HIROMOTO, D.M. Perspectivas no manejo e controle localizado de doenças das culturas: uma abordagem no manejo do nematóide de cisto da soja. In: Borém, A., Giúdice, M.P., Queiroz, D.M., Mantovani, E.C., Ferreira, L.R., Vale, F.X.R. \& Gomide, R.L. (Eds.) Agricultura de Precisão, 2000. pp. 409-453.

TALAMINI, V. Progresso espacial e temporal da Antracnose a partir de diferentes níveis de inoculo inicial em sementes de feijoeiro. 2003. Tese (Doutorado em Fitopatologia) - Universidade Federal de Lavras, Lavras, MG.

TALAMINI, V., POZZA, E.A., MACHADO, J.C. \& OLIVEIRA, F.A. Epidemiologia de doenças associadas a Colletotrichum spp. transmitidas por sementes. Revisão Anual de Patologia de Plantas 10:219-248. 2001.

TAMAYO, P.J. Manejo y control de las enfermedades del frijol voluble (Phaseolus vulgaris L.). Rio Negro. Antioquia, Col., Corporación Colombiana de Investigación Agropecuaria (CORPOICA). Región n ${ }^{\circ}$ 4. Centro de Investigación "La Selva". p.40 (Boletim Técnico)

TANAKA, M.A.S. \& MENTEN, J.O.M. Colletotrichum gossypii e Colletotrichum gossypii var. cephalosporioides em sementes de algodão. Fitopatologia Brasileira 3:125. 1988. (Resumo)

TANAKA, M.A.S. \& MENTEN, J.O.M. Relação entre a resistência do algodoeiro à ramulose e a transmissão de Colletotrichum gossypii var. cephalosporiodes pelas sementes. Summa Phytopathologica 18:227-234. 1992.

WALLACE, M.K. \& HAWKINS, D.M. Applications of geostatistics in plant nematology. Journal of Nematology 26:626-634. 1994.

WEBSTER, R. \& BOAG, B. Geostatistical analysis of cyst nematodes in soil. Journal of Soil Science 43:583-595. 1992. 